\title{
Solid and liquid modernity: A comparison of the social geography of places to die in the UK and Australia.
}

Keywords: Palliative care, Terminal care, Modernity, Social Geography

Dr Duncan Randall ( corresponding author), Principle Teaching Fellow, Health Sciences, University of Southampton, Highfield, Southampton, SO17 1BJ tel: 02380597960 email: d.c.randall@ soton.ac.uk

Dr John P. Rosenberg, Research Fellow, Faculty of Health, Queensland University of Technology, Brisbane, Australia tel: +61 (0)7 31386439 email jp.rosenberg@qut.edu.au

Dr Suzanne Reimer, Associate Professor, Geography and Environment, University of Southampton, Highfield, Southampton SO53 1LJ tel: 02380 598816 email: S.Reimer@soton.ac.uk 
Solid and liquid modernity: A comparison of the social geography of places to die in the UK and Australia.

\begin{abstract}
Preferred place of care and death is a widely used quality measure for palliative and end of life care services. In this paper we explore the use of Zygmunt Bauman's ideas on solid and liquid modernity to understand the complexity of the social geographical contexts of delivering and receiving care. While solid ways of dying offer certainty and standardised care, more liquid ways allow for individualised care connected to family and communities. Understanding the complex tensions between solid and liquid aspects of palliative care may allow practitioners to help dying people to die in the ways and places they prefer.
\end{abstract}

Keywords: Palliative care, Terminal care, Modernity, Social Geography 
LIQUIDITY AND THE SOCIAL GEOGRAPHIES OF DYING

\section{Introduction}

The place of death and the home as an idealised venue for death has become a contested issue in palliative and end of life care (PEoLC; Bluebond-Langner, Beecham, Candy, Langner, \& Jones 2013; Gott, Williams, \& Moeke-Maxwell, 2014; Horsfall, Noonan, \& Leonard, 2012; Rosenberg, 2011). In both the UK and Australia, an expressed preference for home as preferred place of death is identified (Vickers, Thompson, Collins, Childs, \& Hain 2007; Gomes, Calanzani, \& Higginson, 2011; Palliative Care Australia [PCA], 2012) and indeed, a common measure of quality in PEoLC services is congruence between preferred and actual place of death, particularly in the provision of care in domestic dwellings (Burge et al., 2015). These simplistic quality measures of hospital or home can be contrasted with the complexity of delivering and receiving care in different settings. The false dichotomy has evolved based in part on the modern hospice movement's origins as a protest to substandard hospitalised care of dying people (Clark, 2007). It has been suggested that the PEoLC sector has uncritically presumed home is an inherently better place to die (Gott et al., 2014). The histories of PEoLC are shaped by broader social changes affecting understandings of power, certainty and cohesion in the modern world. This suggests a more nuanced understanding of the meaning of place in PEoLC is required (Dyck, Kontos, Angus, \& McKeever, 2005; Rosenberg, 2011).

It is apposite to consider this meaning of place not simply in terms of institutional versus home care, but with reference to the ways in which place is socially constructed in late modernity. We are considering Australia and the UK because both have sophisticated hospital and home nursing systems, and both, while not entirely alike, have PEoLC sectors which provide inpatient care and health care in people's own dwellings (National Institute for Health and Care Excellence, 2015; Leadership Alliance for the Care of Dying People, 2014; PCA, 2012). These two developed, high income, countries have similar but different cultures 
of modernity. The UK has a liberal European culture and Australia, located close to Asia, tends to blend North American influences with aspects of its colonial past. Rather than a comparison of national systems of care, our intention here is to consider the social geographies of nursing care in the two countries. To explore these concepts of social geography and palliative care delivery we have drawn on the work of Zygmunt Bauman (2003, 2005, 2006, 2012), and in particular his thesis of liquid modernity. While Bauman's work has been influential in social cultural studies (Jay, 2010) making it well suited to exploring the cultural aspects of palliative care, Bauman's work has focused less on social geographies and more on the shifts from a historical period of solid modernity to more recent times of liquid modernity.

\section{Dying in Modern Times}

Bauman has written extensively on the concept of modernity (Bauman, 2003, 2005, 2006, 2012) and contributed substantively to the discourse on death and dying (Higo, 2012). While his critics have pointed out that Bauman's work can be difficult to apply and somewhat ethereal (Jay, 2010), his concepts of solid and liquid modernity provide a set of lenses to focus on a disparate set of concerns and issues relating to the social world of dying. Bauman's thesis is based on the premise that at this time in history the social world which was certain, known, ordered, habituated, and resistant to change (i.e., solid) is transforming into an uncertain, unknown, disordered, constantly changing state (i.e., liquid) (Bauman, 2012).

Those who live in a solid mode would aspire to improve through a socially prescribed, known path. By contrast, people living with liquid modernity also aspire to improvement, but established, socially prescribed paths to improvement no longer hold sway. Bauman (2012) describes how liquid modernity is a fractious state, where social anxiety is high. People are 
uncertain about how things work and unsure of what the future will hold. In liquid modernity the old ways, the solid modern ways, dissolve and it is not always clear what will replace these established practices, nor what will result.

We suggest that what we are constructing as a more 'solid' mode of care is present in the medical model of dying. In solid modern dying, one is attended by a medical practitioner who identifies the diagnosis as one which is considered life-limiting, and prescribes a prognosis. Death often occurs in a centralised location which is set aside by the society for the work of dying. In many jurisdictions this is a hospital, although it is uncommon for there to be a discrete space for this purpose; for example, in Australia, of 729 public acute hospitals about 1 in $6(17.7 \%)$ has a hospice or palliative care unit (Australian Institute of Health and Welfare [AIHW], 2014). In the UK, 58\% of deaths occur in hospitals, although there are wide variations between areas between 46\% and 77\% (National Audit Office [NAO], 2008). Death in these settings is controlled and supervised by the health professions (physicians, nurses and others allied to medicine, typically under the direction of a medical practitioner (Johnston \& Smith, 2006). Recipients of this care (patients, carers and families) report varied levels of engagement in the functioning of these places as they go about the business of caring (Johnson \& Smith, 2006), but the structural elements of place are largely solid.

Applied to place and PEoLC, liquid modern dying is decentralised. It occurs in places and spaces not set aside for dying: in people's homes, where they have been living prior to diagnosis. The process of dying is less medically controlled. It may well still involve medical professionals, but partners, parents, other family members, or informal carers play a much larger role and have more control over the setting, and the care delivered and received (Horsfall et al., 2012). Notably, care might be more personalised and more responsive in this liquid state, but also the standards of care can be more variable; home might offer more dignity and privacy, but could leave people with poorly controlled symptoms. 
We argue below that while hospices are centralised location for dying they also have many less solid aspects, such as less supervision by medical professionals, more individualised care. Our exploration follows a pragmatic course, after North American Pragmatics, following the work of people such as Richard Rorty and John Dewey (Murphy, 1990). Specifically we accept Rorty's (1996a) analysis that the best we might be able to achieve is to understand how things hang together at this time and in this cultural political space. Pragmatists reject a universal position that there is a universal truth, in this case a right way to deliver PEoLC which is awaiting our realisation. Rather, they accept a pluralist view that there are many ways to deliver care which may suit particular cultures, at particular times, and places. Rorty sets out how the moral and ethical questions are important, but should be viewed in a frame of such ethical and moral discussion, separately from whether the ideas and practices worked for people at a set time. Thus, for Pragmatists who follow Rorty (1996b) we cannot judge whether PEoLC from a different time was "good" or not; it

may have suited that culture at that time, but not be useful or "good" for us at this time, in our culture. Thus, we do not suggest that either solid or liquid modernity is preferable, but simply that these concepts may help us to understand the ways in which PEoLC is being delivered at this time, in the places and social worlds in UK and Australia.

\section{Solid and Liquid Geographies of PEoLC: A Comparison of UK and Australian Experiences}

To explore the solid (centralised) and the liquid (decentralised) ways of dying we identified three overarching social geographies of micro built environments, service provision, and of power and control spatial geographies. These social geographies of dying are situated within scaled geographies which are local, regional and national (see Figure 1). 


\section{Geographies of Micro Built Environment}

In the UK, the housing stock varies a great deal in age, construction and condition. Hospitals too vary in their age, and design. The design of UK and Australian hospitals has been more to deliver health care than as places for people to die. Thus, the solid aspects of dying in the built environment of the hospital could be contended. However, many hospitals especially more recently have been designed with privacy and dignity in mind offering side room rather than traditional Nightingale wards (Maben et al., 2015). The built environments of the hospice setting perhaps offer the most purposively designed spaces for dying, including features such as private en suite rooms and ceiling rails that allow for people to be hoisted and moved easily into bathrooms. In children's hospices, the construction of snoezelens allow for children disturbed and or anxious to be placed in a calming environment; these rooms are often used with dying children (Hotz et al., 2006). The hospice design and construction may offer then a more solid space for dying than a hospital designed to deliver healthcare resulting in restoration of function.

People's normal places of residence offer a much more liquid built environment. Homes are not normally designed with either healthcare delivery or dying in mind. Common problems include arranging sleeping facilities close to bathrooms and toilets on the same level, lack of storage for medication, equipment and devices and power supplies for equipment. Particularly for elders (but for many others requiring palliative care too), illness and poverty may result in a property not being maintained over an extended period. This can make home settings unsuitable for care delivery either because the state of the property poses a risk to the residents and or to health and social care workers delivering care. Faulty wiring, loose and worn carpeting, broken windows, damp, infestations and faulty heating systems all pose potential dangers which might make the risks of delivering care unacceptable. The built environment of someone's home might be suitable in the palliative care phase, but at the end 
of life an antiquated electrical system might be tipped into failure with the addition of a syringe driver and a feed pump. However, the familiarity of the home environment holds advantages for many people; someone with cognitive impairment may remain continent because they know where the toilet is. Clearly there may be psychological benefits from receiving care in a home setting, in part contributed to by familiarity with the built environment. Buildings designed for the delivery of healthcare and in particular hospices which are often purpose built for PEoLC offer a solid, more certain environment for dying, while the home setting may offer some practical and psychological benefits the environment is more liquid and uncertain.

In surveying a preference for dying at home, then, also asking about the home environment may be useful. The stated preference for dying at home in both states (Vickers et al., 2007; Gomes et al., 2011; PCA, 2012) may be qualified, or reconsidered if the practicalities of receiving care are also considered alongside a statement of preference. We are not suggesting that practicalities of the home built environment will, or should dissuade people from their preference for home, but simply that understanding the social geographies of the built environment and indeed the other geographies explored here can help people to make a informed choice, about where they die, and help all parties to better understand the associated risks of the location of care.

\section{Geographies of Service Provision}

\section{Access and Logistics}

In our analysis, access can be considered as relating to both receiving and delivering care - that is, access for recipients of PEoLC services, and access health and social care workers have to people to deliver PEoLC. In more solid care, access for health and social care workers to deliver care is prescribed and certain. People to whom care is going to be 
delivered are identified by regular medical meetings, ward rounds, or interdisciplinary referrals from identifiable areas of the hospital, such as accident and emergency department, or intensive care environments. Although the UK has a developed hospice movement, palliative care is still a relatively new medical sub-speciality (Clark, 2007). Access to specialist services, consultant palliative care physicians and specialist palliative care nurses remains dependent on local service configuration and, to some extent, on age and the medical condition people are living with; elders living with an oncology condition are much more likely to receive specialist palliative care, despite the fact that children with degenerative neurological conditions also have palliative care needs (Fraser, Miller, Aldridge, McKinney, \& Hain, 2011). There remains a postcode (or State), age and condition lottery as to whether patients, or indeed generalist health and social care workers, can access specialist palliative care services. However, in the UK, with the National Health Service's extensive network of district general hospitals and specialist hospitals, along with the coverage of both adult and children's independent hospices, there is very good access to these more solid, centralised places in which to die.

Access in decentralised, more liquid settings is not so well structured, although the UK has a long and strong tradition of community healthcare and community nursing (Whitting, 2005). In liquid care, the micro aspects of access are much more dynamic and complex; identification of people requiring PEoLC is much less certain. One can easily understand that a person living with a life-limiting condition may deteriorate unnoticed, or that informal carers may not know what signs to look for, or may ignore signs for various reasons. In addition, those who require care can be located anywhere in the geographical area covered by healthcare teams. The rural landscape and population density makes delivering care to some communities challenging - particularly in Australia - but urban areas can hold their own challenges to gaining access. For example, while delivering PEoLC in the islands off 
northern Scotland or in remote farmland in western Queensland may be restricted by the travelling time between people, in London or Sydney healthcare workers can find it difficult to find a place to park their car or be prevented from travelling at certain times by traffic congestion. Thus, access for healthcare workers to deliver care is more complex and less prescribed. One set of traffic works and access to deliver care at home can be made virtually impossible. However, more liquid settings do allow people greater access to their family, friends and communities, and for these groups to access the dying person (Horsfall, Yardley, Leonard, Noonan, \& Rosenberg, 2015). Community access may be important in delivering culturally safe care, particularly for aboriginal Australians (O’Brien et al., 2013).The ease of access staying at home or dying in a local hospice can afford can reduce a sense of isolation and may help carers and other family members accept death, which may facilitate grieving (O’Brien et al., 2013).

Access may be curtailed by too many competing demands on a team of healthcare workers, located in different parts of a geographical area. However, such peak demands on teams may be rare and, as such, this might partly explain why, despite a good infrastructure, new technologies such as telemedicine have not been widely adopted in the UK. The geographical scale of the UK means that often one can travel and meet with people in person. Thus, the access to specialist palliative care workers has not developed into telemedicine because more often than not a visit in person is possible, albeit that such person-to-person visits are more expensive and time- and resource-intensive. Another explanation might be the poor track record of the NHS with electronic/online projects (Papoutsi et al., 2015). Notably, the use of telehealth for PEoLC in such a vast and sparsely populated place as regional, rural and remote Australia has remained very low despite the technical possibilities and the geographical isolation. 
Access in solid care settings is then more predictable and certain while in the liquid state access is complex and less predictable. To a certain extent, logistics and access issues overlap. However, there are separate logistical aspects of delivering care in solid and liquid settings. There are the logistical aspects of staffing and expertise alluded to above (where people may not be able to access specialist care because of a lack of staff for some groups e.g., children in some areas, Carter et al., 2012). The logistics of medical equipment, devices and medications pose quite different set of challenges. In solid care the logistics of care equipment and medication may seem to almost disappear, because they have become routine and regimented so as to become habitual. To a certain extent this also holds for hospice care, although often they do not have the economies of scale that a hospital may enjoy and often do not have the access to central services, such as medical equipment departments.

However, in liquid decentralised dying logistical issues raise significant challenges. Some households are not equipped to cope with a dying person. At a simple level the washing machine which might have coped with the regular family washing may break under the strain of near constant use doing the washing for an incontinent person. In rural areas a local pharmacy may be difficult to access and may not carry the wide range of preparations and medication required to deliver end of life care. Although this is usually a temporary problem and community pharmacist and pharmacies usually provide an excellent service in the community. In more liquid care the food preferences for different cultural groups is much easier to accommodate than where catering is supplied by a central hospital kitchen. Catering problems can often be solved by the use of online shopping from major supermarkets now available in many areas, or the extensive take away restaurant delivery services available in most areas in the UK and Australia, especially in urban and suburban areas.

Medical equipment and devices pose more of a challenge. The difficulties of ensuring the supply of disposable devices, such as feeding reservoirs and incontinence devices is often 
cited by carers as one of the most frustrating aspects of delivering care at home (Kirk, Glendinning, \& Callery, 2005). The challenges of ensuring appropriate equipment is available as required as people's needs change in PEoLC are compounded by the lack of medical engineering support in many community health services. Thus, it can be more challenging to obtain a syringe driver in community settings to deliver pain relief, and one may be less certain about when the syringe driver was last serviced or calibrated. The logistics of delivering care in more liquid community settings are fraught with many challenges, not often evident in the solid care delivered in hospitals with centralised established services which employ economies of scale. The geographies access and logistics allow for a detailed consideration of the provision of care in different locations, including a risk assessment of the capacity to deliver care and the safety of workers delivering care.

\section{Geographies of Power and Control}

In solid dying, the power and control rests more with medical and other health and social care professionals than with the person dying or their representatives (family/communities). Hospitals and hospices are organised and sustained by health care professionals. To some extent recent trends in health care in which a patient, public involvement agenda has come to the fore have altered the dominance of professionals. However, the degree to which such a "public" are allowed to be independent is debatable (Gibson, Lewando-Hundt, \& Blaxter, 2011). In terms of dying in hospital, medical professionals control the access to medication and all other services and can call on hospital security services to enforce their decisions. The control and power of medical practitioners and other professionals is somewhat more liquid in the hospice setting where service users, religious groups and to some extent other community leaders have a much stronger voice. Hospices do not normally have their own security staff in the same way most hospitals do. 
In the liquid setting of home care, power and control is substantially shifted to the person receiving care and their carers. Health professionals' role in home care has been described as one of a guest in the house (Rosenberg, 2011). Social norms are often enacted such as seeking permission to use facilities and carers offering refreshment (tea or coffee). While health and social care professionals' social standing should not be underestimated in community settings, people may continue to respect the views and follow the instruction of a professional because of their professional status even in their own home (Kirk et al., 2005). It remains evident that people receiving care and their carers can much more easily refuse care and alter delegated care - including medication - in their own homes than they can in a hospital setting. In the liquid decentralised setting, even this shift of power and control can be contended (Kirk et al., 2005). Health professionals continue to control the legal and practical access to opiate medication, and to most medical equipment and devices. They can also call on State courts and police forces to have a dying person removed to a hospital if they suspect abuse or neglect. The liquid setting of the home may provide people with more control over their death, and it may be easier for people and carers to adapt care to meet their own needs, for example delivering a more flexible approach to symptom management freed from the strictures of delivering care to a group in the structures of the hospital or hospice. However, this power and control comes at a cost, being separate from the hospital or hospice it can be more difficult to engage health care professionals, and there is always the threat that some choices might be interpreted as neglect or abuse by professionals and the sanctions of the State imposed. Much of this argument on power and control is speculative as empirical evidence on how power is enacted in home care is an area for research development, and none has been framed by Bauman's concept of anxiety arising from liquid modernity.

A critique which might be offered is that the solidity lens does not account for gendered politics of caring. The more person centred liquid dying is only possible if the person is 
supported by a carer and often this role falls to women (Brown, 2004). It might be suggested that the division between solid and liquid could also be expressed as a continuum of objective rational masculine approaches and subjective, intuitive feminine ones. That what is at stake here is merely semantics. However, the frame of modernity would seem to have some differences as opposed to a frame of gendered social geographies. The anxiety Bauman (2012) describes associated with liquid modernity perhaps explains the shifts between more solid and more liquid service offers. Typically people offered a home care package are often admitted to hospital during late end of life care. Understanding the anxiety, that the more liquid person centred home care might engender, explains why, despite the best efforts of community nurses, people and their carers might switch to a hospital. In addition, it should allow such switching to be understood, not as a failure of care, but as a process whereby the person and/or their carer seek the certainty they associate with the more solid form of hospital care. It may also help to explain why people may be upset when the care offered in hospital does not provide the solid certainty that was expected, or the frustration people may feel in losing the person centred liquid death that they had hoped for. Thus, using Bauman's ideas allows a much more nuanced and complex analysis of how the perceptions of care may affect both those who die and their carers, much more than the gendered politics of care can offer, although the gendered perspectives of solid and liquid modernity should be considered and explored in more depth.

\section{Cultural Aspects}

It can be challenging for health and social care professionals in multicultural and divided societies such as the UK and Australia to offer a solid version of dying in centralised location such as hospitals and hospices. Such societies contain various communities with their own cultures and religious practices relating to dying and death. The standard response to this challenge has been to espouse equality, liberal doctrine, where it is claimed everyone 
receives equal treatment and consideration. However, we know that people from ethnic minorities have very different experiences of PEoLC (Broom, Good, Kirby \& Lwin, 2013) as do people with learning difficulties (Dunkley \& Sales, 2014). The solid centralised palliative care delivery of course makes meeting all needs from different communities often impossible as the group of people receiving care at any one time may come from different communities with different and occasionally conflicting psychosocial and cultural needs. For example Arabic cultures generally favour large family gatherings at the bedside of a dying person, but Western nursing practice restricts visitor numbers and often limits visitor time. Moreover Western traditions favour few people at the bedside and value privacy and quiet. This is equally the case in parts of regional, rural and remote Australia where the provision of inpatient PEoLC for indigenous Australians cannot always meet the particular needs of the extended networks fundamental to traditional notions of family (O'Brien et al., 2013). Often the official rules of the hospital which are usually designed for curative care are ignored or flouted for palliative patients and their families, but the cultural practices remain illegitimate and marginalised. The solidity of modern dying tends then to offer a one size to fit all, with some covert flexibility.

Dying at home might be seen as offering a much more individualised and personal experience. The liquid setting of community care offers a much more individually tailored care. Generally speaking, one person is dying at any one time in a household (even if a household were particularly afflicted the same social and cultural practices in relation to dying and death would apply). This might account for why in most surveys home is stated as the preferred place for PEoLC. It is perhaps not a surprise that in highly individualised societies such as Australia and the UK that people would choose an obviously more individualised care delivery setting. The stereotypical cultural traps of home care are perhaps well known (Gott et al., 2014), although assumptions about cultural cohesion and adherence 
may still result in poor, or no care provision (Broom et al., 2013). In addition, there are challenges of over medicalising the home setting, the dangers of an over romantic view of home, which may be the site of abuse or failed relationships (Gott et al., 2014; Fisher, 2003). For some a death at home can offer control, dignity and the opportunity for individual, family and community preparation for death (Horsfall et al., 2015). However, for others it may feel like being abandoned to be cared for by people, with whom relationships have become marked by resentment or fear. There is also an assumption that people will have family members, or others, capable and willing to provide care. How health and social care workers provide care in the era of individual, personal care when dying people do not have a carer and are reliant on professional care seems problematic. Using Bauman's liquid modernity may help explore how responsibility can be taken for caring for people without taking responsibility away from dying people themselves.

The perception of hospitals and hospices as places where people die and or are cared for prior to death is at odds with the desire of many people to die at home. Although the statistics show, in both Australia and the UK, that the majority of people do in fact die in hospitals (with some considerable geographical variations within each country, see NAO, 2008; AIHW, 2014). The solid modern scientific death offered in hospitals may be less individualised, but the liquid home death, while more culturally safe and individualised, can be subject to professional assumptions which affect care practices and quality and which in some cases leave people at risk of neglect or abuse.

\section{Conclusions}

In utilising Bauman's thesis of solid and liquid modernity, we can see that the solidity of some PEoLC services is found in the built environments and organisation of hospitals and, to a lesser extent, hospices. The predominance of the medical model in health care promotes hierarchical and procedural clarity that might seem to offer a less personal, less culturally 
safe approach to dying, but provides a more standardised approach, more certain access to medication, equipment, devices and health and social care expertise. Conversely, liquid settings offer more control and power to people receiving palliative care and their carers, with the engagement of informal networks of care. They may offer more individualised and culturally sensitive practices of dying, but access to medication, equipment and health and social care expertise is less certain.

In places in our argument above we may have given the impression that more solid dying occurs in hospitals, or hospices and more liquidity in dying is achieved in home settings. However, PEoLC is delivered and received in a nexus of settings and may shift for an individual between hospice, home, and hospital. In addition, institutions may design and deliver PEoLC which is more solid or more liquid. Hospice care provides examples of how care might in the terms set out in this paper be both solid and liquid. Hospices are recognised as formal setting in which to die in individual ways, equally as argued above a hospital may provide a person centred more liquid way of dying. Thus, for both institutions and individuals, PEoLC may vary between solid and liquid states.

A potential challenge to the use of Bauman's ideas might be that his work is relativist. As argued above at times PEoLC care can seem to be both solid and liquid, as if all care was a gel. If, as we have stated, neither the solid nor the liquid modernity approach to PEoLC is valued as one above the other, then are they not both equally of use? As we have argued PEoLC or certain aspects of it may be situated along a continuum. Rarely, if ever does an element sit wholly on the solid or liquid end of the continuum. The fact that sometimes in certain cases the PEoLC can seem gel like, both liquid and solid, does not negate the fact that certain aspects might be located further to the solid or liquid end of the continuum. The occasions when care might be situated in the centre of the continuum does not reduce the continuum to a single point. As stated above as we take a Pragmatist view of this work 
relativism and context is embraced rather than avoided (Rorty 1996a). However, being aware of the solid-liquid continuum could help both health and social care workers and people receiving care to examine the benefits of a location and understand the risks. In addition, understanding that, while liquid dying might be a stated preference (expressed as dying at home), it is accompanied by anxiety at the uncertainty associated with the liquid state. This could allow health and social care workers to address such anxiety and provide interventions to help people cope with the anxiety. Alternatively, policy makers and health and social care managers could look at these factors of the liquid state and design and deliver polices to reduce the uncertainty in home care provision. An example might be investing in medical equipment services, Pharmacy and expert palliative care clinicians to ensure 24 hour, 7 days a week cover in communities.

In both countries, urbanity and rurality play a major role in the provision of PEoLC. Whilst the distances involved health care service provision in rural UK and rural Australia are worlds apart, the impact of relative distance of home to services results in similar challenges to symptom management, carer support and achieving a home death. Moreover, rural communities may undertake local cultural and community practices to support their dying members that are less prevalent in urban centres. Further work could improve our understanding of urbanity and rurality in relation to dying in home, hospice and hospital settings, which might include understanding the social capital and cultural community aspects as well as designing and evaluating interventions tailored for the urban and rural environments.

This conceptual analysis suggests that both solid and liquid modernity modes of delivering and receiving PEoLC carry advantages and challenges. The focus of health and social care policy and practice then should not be on either solid or liquid ways of dying, but on understanding how health and social care practice might solidify and liquefy in different 
contexts and circumstances. Such that professionals and carers and those receiving PEoLC understand the challenges and advantages of more, or less solid ways of delivering and receiving care. The preferred place of death measure used by many as a quality measure might then be replaced with more subtle and useful assessments of whether people want and can cope with more personal flexible liquid care or more solid PEoLC. In turn, these degrees of liquidity or solidity may have an effect on dying and bereavement. Finally this new analysis would allow for the anxiety which might be provoked by less certain liquid practices to be addressed and ameliorated, while the lack of personalisation in more solid settings might be reduced without losing the sense of security more solid care settings can offer.

\section{Funding}

This research received no specific grant from any funding agency in the public, commercial, or not-for-profit sectors. 
Figure 1: Solid- Liquid Dying in Social Geographies

Scaled Geographies:

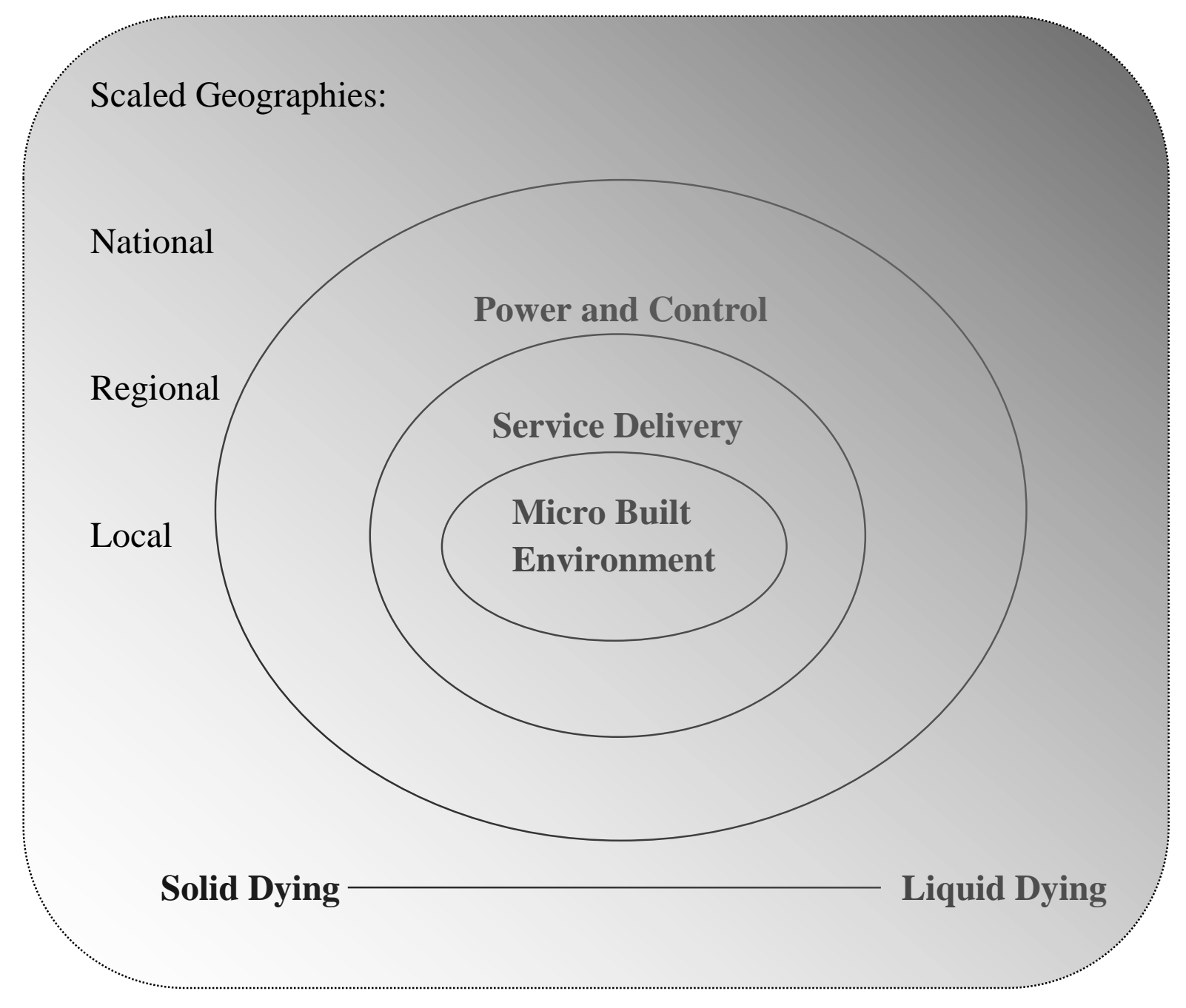




\section{References}

Australian Institute of Health \& Welfare. (2014). Australian hospital statistics 2012-13. Canberra, Australia: Author.

Bauman, Z. (2003). Liquid love. Cambridge: Polity Press.

Bauman, Z. (2005). Liquid life. Cambridge: Polity Press.

Bauman, Z. (2006). Liquid fear. Cambridge: Polity Press.

Bauman, Z. (2012). Liquid modernity. Malden, MA: Polity Press,

Bluebond-Langner, M., Beecham, E., Candy, B., Langner, R., \& Jones, L. (2013). Preferred place of death for children and young people with life-limiting and life-threatening conditions: A systematic review of the literature and recommendations for future inquiry and policy. Palliative Medicine, 27, 705-713. doi:10.1177/0269216313483186

Broom, A., Good, P., Kirby, E., \& Lwin, Z. (2013). Negotiating palliative care in the context of culturally and linguistically diverse patients. Internal Medicine Journal, 43, 10431046. doi:10.1111/imj.12244

Brown, M. (2004). Between neoliberalism and cultural conservatism: Spatial divisions and multiplications of hospice labour in the United States. Gender, Place and Culture,11, 67-82. doi:10.1080/0966369042000188558

Burge, F., Lawson, B., Johnston, G., Asada, Y., McIntyre, P.F., \& Flowerdew, G. (2015). Preferred and actual location of death: What factors enable a preferred home death? Journal of Palliative Medicine, 18, 1054-1059. doi:10.1089/jpm.2015.

Carter, B., Coad J., Bray L., Goodenough T.,Moore A., Anderson C., Clinchant, A., \& Widdas, D. (2012). Home-based care for special healthcare needs: Community children's nursing services. Nursing Research, 61, 260-268. doi:10.1097/NNR.0b013e31825b6848

Clark, D. (2007). From margins to centre: A review of the history of palliative care in cancer. Lancet Oncology, 8, 430-38. doi:10.1016/S1470-2045(07)70138-9

Dunkley, S., \& Sales, R. (2014). The challenges of providing palliative care for people with intellectual disabilities: a literature review. International Journal of Palliative Nursing, 20, 279-284. doi:10.12968/ijpn.2014.20.6.279

Dyck, I., Kontos, P., Angus, J., \& McKeever, P. (2005). The home as a site for long term care: Meanings and management of bodies and spaces. Health and Place, 11, 173-85. doi: 10.1016/j.healthplace.2004.06.001

Fisher, C. (2003). The invisible dimension: Abuse in palliative care families. A review. Journal of Palliative Medicine, 6, 257-264.doi:10.1089/109662103764978515

Fraser, L. K., Miller, M., Aldridge, J., McKinney, P. A., \& Hain, R. (2011). Life-limiting and life-threatening conditions in children and young people in the United Kingdom; national and regional prevalence in relation to socioeconomic status and ethnicity. Bristol: Division of Epidemiology, University of Leeds and Together for Short Lives. 
Gibson, A. J., Lewando-Hundt, G., \& Blaxter, L.(2011). Weak and strong publics: Drawing on Nancy Fraser to explore parental participation in neonatal networks. Health Expectations, 17, 104-115. doi: 10.1111/j.1369-7625.2011.00735.x

Gomes, B., Calanzani, N., \& Higginson, I. J. (2011). Local preferences and place of death in regions within England 2010. London: Cicely Saunders International.

Gott, C. M., Williams, L., \& Moeke-Maxwell, T. (2014). The paradoxes of 'home' within a palliative care context. In M. Roche, J. Mansvelt, R. Prince, \& A. Gallagher (Eds.), Engaging geographies: Landscapes, lifecourses and mobilities (pp. 137-151). Cambridge: Cambridge University Press.

Higo, M. (2012). Surviving death-anxieties in liquid modern times: Examining Zygmunt Bauman's cultural theory of death and dying. Omega: Journal of Death and Dying, 65, 221-238. doi:10.2190/OM.65.3.e

Horsfall, D., Noonan, K., \& Leonard, R. (2012). Bringing our dying home: How caring for someone at end of life builds social capital and develops compassionate communities. Health Sociology Review, 21, 373-382.doi: 10.5172/hesr.2012.21.4.373

Horsfall, D., Yardley, A., Leonard, R., Noonan, K., \& Rosenberg, J. (2015). End of life at home: Co-creating an ecology of care. University of Western Sydney/Cancer Council NSW/Calvary Centre for Palliative Care Research (Australian Catholic University)/Queensland University of Technology/CSIRO.

Hotz, G. A., Castelblanco, A., Lara, I. M., Weiss, A. D., Duncan, R., \& Kuluz, J. W. (2006). Snoezelen: A controlled multi-sensory stimulation therapy for children recovering from severe brain injury, Brain Injury, 20, 879-888.doi: 10.1080/02699050600832635

Jay, M. (2010). Liquidity crisis: Zygmunt Bauman and the incredible lightness of modernity. Theory, Culture and Society, 27(6), 95-106.doi: 10.1177/0263276410382024

Johnston, B., \& Smith, L. N. (2006). Nurses' and patients' perceptions of expert palliative nursing care. Journal of Advanced Nursing, 54), 700-709.doi:10.1111/j.13652648.2006.03857.x

Kirk, S., Glendinning, C., \& Callery, P. (2005). Parent or nurse? The experience of being the parent of a technology-dependent child. Journal of Advanced Nursing, 51, 456464.doi:10.1111/j.1365-2648.2005.03522.x

Leadership Alliance for the Care of Dying People. (2014). One chance to get it right: improving people's experience of care in the last few days and hours of life. Retrieved from https://www.gov.uk/government/uploads/system/uploads/attachment_data/file/323188/ One_chance_to_get_it_right.pdf

Maben, J., Griffiths, P., Penfold, C., Simon, M., Pizzo, E., Anderson, J., Robert, G., Hughes, J.,, Murrells, T., Brearley, S., \& Barlow, J. (2015). Evaluating a major innovation in hospital design: Workforce implications and impact on patient and staff experiences of all single room hospital accommodation. Health Services and Delivery Research, 3(3), 1-304. doi:10.3310/hsdr03030

Murphy, J, (1990). Pragmatism: From Peirce to Davidson. Boulder, CO: Westview Press, Oxford. 
National Audit Office. (2008). End of life care. London: The Stationery Office. Retrieved from https://www.nao.org.uk/wp-content/uploads/2008/11/07081043.pdf

National Institute for Health \& Care Excellence. (2015). Care of dying adults in the last days of life (NG31) Retrieved from https://www.nice.org.uk/guidance/ng31

O'Brien, A. P., Bloomer, M. J., McGrath, P., Clark, K., Martin, T., Lock, M., Pidcock, T., van der Riet, P., \& O'Connor, M. (2013). Considering Aboriginal palliative care models: The challenges for mainstream services. Rural and Remote Health 13: 2339. (Online) Retrieved from http://www.rrh.org.au/publishedarticles/article_print_2339.pdf

Palliative Care Australia. (2012). Submission to the Australian Senate Standing Committee on community affairs inquiry into palliative care in Australia. Canberra, Australia: Author.

Papoutsi, C., Reed, J. E., Marston, C., Lewis, R., Majeed, A., \& Bell, D. (2015). Patient and public views about the security and privacy of Electronic Health Records (EHRs) in the UK: Results from a mixed methods study BMC Medical Informatics and Decision Making, 15:86. doi:10.1186/s12911-015-0202-2

Rorty, R. (1996a). Introduction: Relativism: Finding and making. In R. Rorty (Ed.), Philosophy and social hope (pp.xvi-xxxii). London: Penguin.

Rorty, R. (1996b). Wild Orchids and Trostky. In R. Rorty (Ed.), Philosophy and social hope (pp 3-22). London: Penguin.

Rosenberg, J. P. (2011). Whose business is dying? Death, the home and palliative care. Cultural Studies Review, 17(1), 15-30. doi:10.5130/csr.v17i1.1971

Vickers, J., Thompson, A., Collins, G. ., Childs, M., \& Hain, R. (2007). Place and provision of palliative care for children with progressive cancer: A study by the Paediatric Oncology Nurses' Forum/United Kingdom Children's Cancer Study Group Palliative Care Working Group. Journal of Clinical Oncology, 25, 4472-4476. doi:10.1200/JCO.2007.12.0493

Whitting, M. (2005). 1888-2004: A historical overview of community children's nursing. In A. Sidey \& D. Widdas (Eds.), Textbook of Community Children's Nursing (2 ${ }^{\text {nd }} \mathrm{ed} ., \mathrm{pp}$. 17-41). Edinburgh: Elsevier. 